\title{
Nanoscale Compositional Characterization of Silicon Oxide-Nitride-Oxide Stacks
}

Igor Levin

Ceramics Division, National Institute of Standards and Technology, Gaithersburg MD 20899

Silicon oxynitrides are the leading candidates to replace pure thermal $\mathrm{SiO}_{2}$ as a gate dielectric; oxynitride films provide superior suppression of boron penetration from the poly-Si gate, enhanced reliability and reduced hot-electron induced degradation [1]. At the same time, silicon oxide-nitrideoxide multilayers (ONO stacks) attracted considerable interest for the charge storage structures in

non-volatile memory devices. The electrical performance of both the oxynitride- and ONO-based devices is strongly affected by the depth distribution of nitrogen atoms; however, few systematic studies of elemental distributions in ONO stacks have been reported [2]. Electron energy loss spectroscopy (EELS) in a transmission electron microscope offers a unique combination of the (i) high sensitivity to both oxygen and nitrogen, (ii) high spatial resolution (1 $\mathrm{nm}$ or better) and (iii) ability to acquire EELS spectra from specific locations selected in the TEM image [3]. Additionally, analysis of both chemical shifts of the characteristic absorbtion edges and details of the near-edge structure in the EELS spectra can provide useful information on the atomic bonding. Therefore, in the present work, we applied spatially resolved EELS to analyze elemental distributions in the ONO stacks as a function of processing conditions.

The ONO stacks for this study were processed at Tower Semiconductor Ltd. (Israel). The stacks consisted of a thermally grown bottom oxide $(5-6 \mathrm{~nm})$, an LPCVD deposited silicon nitride layer (6$7 \mathrm{~nm})$, and a top oxide layer formed either by nitride reoxidation or TEOS deposition $(8-13 \mathrm{~nm})$. Both spatially-resolved EELS measurements and structural imaging were conducted in a JEOL-3010 UHR (300 kV, thermo-ionic $\mathrm{LaB}_{6}$ source) HRTEM equipped with a GATAN Imaging Filter (GIF). The EELS experiments were conducted as following:

1) The HRTEM image of the ONO stack was rotated (by adjusting an excitation of the projector lens) so that the interfaces were set parallel to the energy-dispersion direction of GIF.

2) The EELS spectra containing characteristic $\mathrm{Si}_{2,3}, \mathrm{O}-\mathrm{K}$ and $\mathrm{N}-\mathrm{K}$ edges were recorded with the energy dispersion of $0.1 \mathrm{eV} / \mathrm{ch}$. The spectra as recorded with a CCD camera have two-dimentions: horizontal, which represents energy loss, and vertical, which represents spatial coordinate. Therefore, each 2-D spectrum recorded with CCD contains series of EELS spectra recorded across the ONO stack; that is, such 2-D spectrum represents a spectrum-line. The spatial resolution in this method is limited predominatly by (i) specimen drift during acquistion, (ii) chromatic aberration of the objective lens. Under the presently used experimental conditions, the spatial resolutions of 0.8-1 nm were obtained.

3) The spectrum-lines were processed to remove background and, the intensity under each edge was integrated over selected energy window (typically 1-2 eV). Finally, the distributions of integrated intensity as a function of spatial coordinate were obtained.

Typical HRTEM image of the ONO stack along with the corresponding $\mathrm{O}-\mathrm{K}$, and $\mathrm{N}-\mathrm{K}$ integrated intensity profiles are shown in Fig. 1 and 2, respectively. The nitride layer appears dark in the image, consistent with its density being higher than those of the oxide layers, as measured by xray reflectometry. The nitrogen distribution reproducibly exhibited small peaks both at the $\mathrm{Si} / \mathrm{SiO}_{2}$ interface and at the outer surface of the stack; the results were qualitatively similar for all ONO specimens investigated. Analyses of the $\mathrm{Si} / \mathrm{SiO}_{2} / \mathrm{Si}_{3} \mathrm{~N}_{4}$ specimen (without top oxide) revealed presence of similar nitrogen peak at the $\mathrm{Si} / \mathrm{SiO}_{2}$ interface, which indicates that the nitrogen segregation occurred during LPCVD deposition of a nitride layer. According to our results, subsequent growth (or deposition) of the top oxide layer had no significant effect on the nitrogen segregation to the $\mathrm{Si} / \mathrm{SiO}_{2}$ interface; however, additional segregation of nitrogen to the outer surface of the top oxide was observed. 


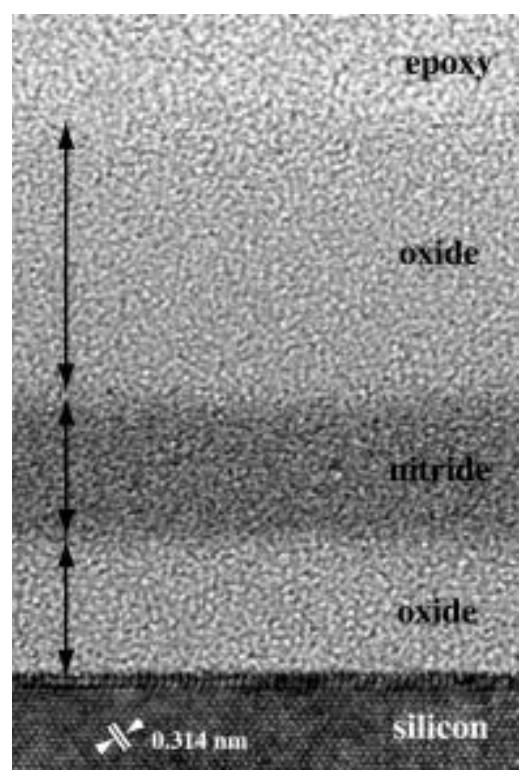

Fig. 1: HRTEM image of the ONO stack

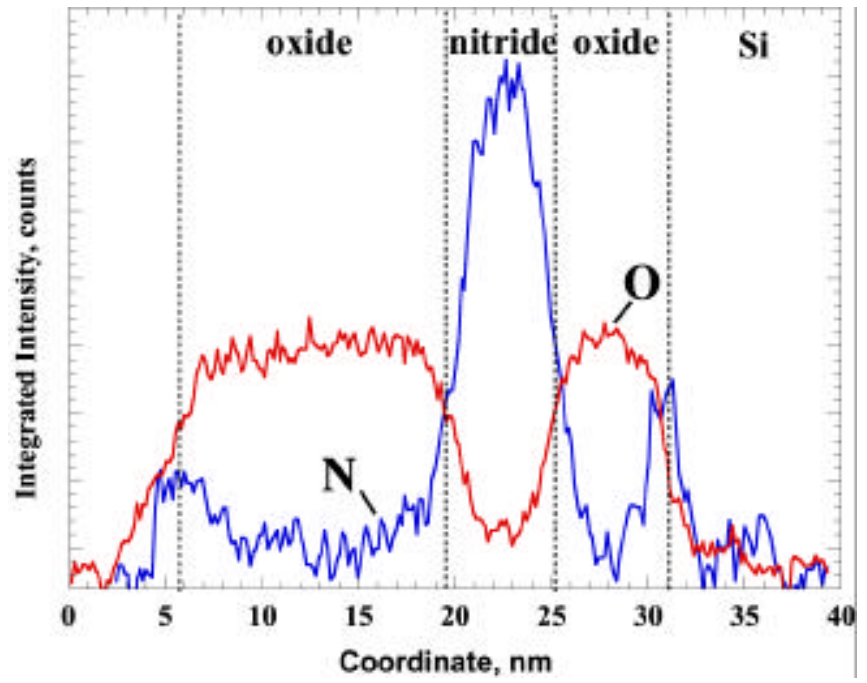

Fig. 2: O-K and N-K integrated intensity distribution profiles across the ONO stack.

References:

[1] G. Lukovsky, IBM J. Res. Develop., 43 (3) (1999) 301-326

[2] E.P. Gusev et al., IBM J. Res. Develop., 43 (3) (1999) 265-286

[3] K. Kimoto et al., Micron 30 (1999) 121-127 